\title{
Risk assessment for ancillary services
}

\author{
Omer Hadzic ${ }^{1}$, Smajo Bisanovic ${ }^{2}$ \\ ${ }^{1}$ Independent System Operator in Bosnia and Herzegovina, Bosnia and Herzegovina \\ ${ }^{2}$ Public Enterprise Elektroprivreda of Bosnia and Herzegovina d.d.-Sarajevo, Bosnia and Herzegovina
}

\begin{tabular}{|c|c|}
\hline Article Info & ABSTRACT \\
\hline Article history: & \multirow{7}{*}{$\begin{array}{l}\text { The power trading and ancillary services provision comprise technical and } \\
\text { financial risks and therefore require a structured risk management. Focus in } \\
\text { this paper is on financial risk management that is important for the system } \\
\text { operator faces when providing and using ancillary services for balancing of } \\
\text { power system. Risk on ancillary services portfolio is modeled through value } \\
\text { at risk and conditional value at risk measures. The application of these risk } \\
\text { measures in power system is given in detail to show how to using the risk } \\
\text { concept in practice. Conditional value at risk optimization is analysed in the } \\
\text { context of portfolio selection and how to apply this optimization for hedging } \\
\text { a portfolio consisting of different types of ancillary services. }\end{array}$} \\
\hline Received Nov 11, 2018 & \\
\hline Revised Nov 20, 2018 & \\
\hline Accepted Dec 19, 2018 & \\
\hline Keywords: & \\
\hline Ancillary services & \\
\hline Conditional value at risk & \\
\hline
\end{tabular}

Copyright (C) 2019 Institute of Advanced Engineering and Science. All rights reserved.

\section{Corresponding Author:}

Smajo Bisanovic,

Public Enterprise Elektroprivreda of Bosnia and Herzegovina d.d.-Sarajevo,

Vilsonovo setaliste 15 ,

71000 Sarajevo, Bosnia and Herzegovina.

Email: s.bisanovic@epbih.ba

\section{INTRODUCTION}

In an energy-economical context, it is necessary to discuss risk management in relation to two different but closely related aspects: the electricity market and the ancillary service provision. In the context of the electricity market, risk management differs from short-term and long-term trading. In both trading activities, risk management is important and is already used in companies. The most important risks are the price risk and the risk of not being able to produce the sold energy to a contract partner. Another aspect of risk management is concerned with ancillary service provision. Ancillary services, such as frequency and voltage control, are required for a reliable and stable power supply. Regarding for example secondary control reserve, power units can sell a constant power reserve to the operating reserve market, realising a variable price that orientates itself on the bid of the unit owner. This power reserve will be activated when occured imbalance or when the frequency is higher or lower than the nominal value. The uncertainty of the surplus or deficit power production yields the risk of a retribution payment to the operating reserve market [1].

Ancillary services in the power system in any country of the world are important for overall security and reliability of the system. Their importance is contained within the process of supporting the basic functions of the system (active power generation, electricity supply) and they are provided by subjects responsible for generation, transmission, distribution and control (control+regulation) of the system. An important characteristic of ancillary services in the power system is to provide at any moment their availability in terms of their capacity/quantity. Therefore the costs of ancillary services are defined as opportunity-costs or as costs of undelivered active power in the power system in an amount in which specific ancillary service is to be ensured [1].

It is very important to determine costs for each of the ancillary services since today for most of them there are no adequate and available-reliable data. In addition, it is important, in techno-economic and legal terms, to explore the issue of ensuring ancillary services in the market environment such as: liability to 
provide ancillary services, providing ancillary services on the contract basis (for example, by a periodical tender announcement), procurement of ancillary services in the market (for example, through the initial bid market of ancillary services, where is initial bid market - market with set initial prices).

For the system operator it is important to have some knowledge of its risk exposure. Risks can come from uncertainty in markets, project failures, legal liabilities, accidents and natural causes. So the risk management is the identification, assessment and prioritization of risks (by risk measurements) followed by coordinated and economical application of resources to minimize, monitor and control the probability and/or impact of unfortunate events (by risk treatments) [2]. Market risk is a complex subject with multiple dimensions and implications for electricity business activity. Analytical risk measures such as traditional Greek measures or high-order and cross-sensitivities allow us to control in detail market risk, but sometimes these analytical risk measures are too technical to be understood by non-technical staff or by management [3]. Hence, it is necessary to make a synthesis of the information contained in analytical measures into a more intelligible form. The natural way of creating such a type of risk measure is that of assessing the impact of risky events in monetary terms, because non-technical people are also capable of understanding the meaning of a potential monetary (or economic) loss. This is exactly the reason why synthetic risk measures have been introduced and have reached a very high importance in the last ten years [1]. A typical example is represented by Value at Risk, but this indice is not the only synthetic risk measure important to consider in the electricity field, especially if the business we are interested to riskassess is not completely based on financial trading [3-5].

In this paper risk measures Value at Risk and Conditional Value at Risk are used for the assessment risk in the ancillary services provision. At the beginning of the procedure to specify a risk measure, one has to determine a probability distribution function of the variable of interest, where a normally distributed function can be assumed. This assumption is often justified in a financial context. In order to find the right parameters of the distribution function, for instances mean value and standard deviation for a normal distribution, one has to have some data about the variable. One possibility is to take historic values of the variable and/or its frequency of occurrence. The second possibility is to simulate the problem (in MonteCarlo simulation) and extract out of these results the needed data $[6,7]$.

This paper is organized as follows. Section 2 provides the mathematical framework for risk measures such as Value at Risk and Conditional Value at Risk. In Section 3 the results are presented and discussed. The conclusions and point to future research are outlined in Section 4.

\section{VALUE-AT-RISK AND CONDITIONAL VALUE-AT-RISK AS A RISK MEASURES}

In this chapter the mathematical definitions of Value at Risk and Conditional Value at Risk are given, followed by an intuitive description of their properties and interactions.

\subsection{Value at Risk}

Value at Risk $(\mathrm{VaR})$ is the maximum loss $c$ that will not be exceeded at a given confidence level $\alpha$. For a given portfolio, probability distribution, probability level and time horizon, $\mathrm{VaR}$ is defined as a threshold value such that the probability that the loss on the portfolio, based on (fair) market prices without trading in the portfolio and over the given time horizon, exceeds this value is the given probability level. This gives the following mathematical definition of $\operatorname{VaR}[8,9]$.

\section{Let $X$ be a random variable representing loss. Given parameter $0<\alpha<1$, the $\alpha$-VaR of X is:}

$$
\operatorname{VaR}_{\alpha}(X)=\min \{c: P(X \leq c) \geq \alpha\}
$$

VaR can have several equivalent interpretations [9]:

- $\operatorname{VaR}_{\alpha}(\mathrm{X})$ is the minimum loss that will not be exceeded with probability $\alpha$;

- $\operatorname{VaR}_{\alpha}(\mathrm{X})$ is the $\alpha$-quantile of the distribution of $\mathrm{X}$;

- $\operatorname{VaR}_{\alpha}(\mathrm{X})$ is the smallest loss in the $(1-\alpha) \times 100 \%$ worst cases;

- $\operatorname{VaR}_{\alpha}(\mathrm{X})$ is the highest loss in the $\alpha \times 100 \%$ best cases.

Suppose the current price of a portfolio is $\mathrm{P}_{0}$ and the rate of return $\mathrm{R}$ for this portfolio is normally distributed with mean $\mu$ and standard deviation $\sigma$. Then the portfolio value at the end of the time horizon is $\mathrm{P}_{1}=(1+\mathrm{R}) \times \mathrm{P}_{0}$ with mean $(1+\mu) \times \mathrm{P}_{0}$ and standard deviation $\sigma \mathrm{P}_{0}$. Firstly, we denote the lowest portfolio value at some confidence level $\alpha$ as $\mathrm{P}^{*}{ }_{1}=\left(1+\mathrm{R}^{*}\right) \times \mathrm{P}^{*}{ }_{0}$. Then the VaR number relative to the expected return is $\operatorname{VaR}($ mean $)=\mathbf{E}\left[\mathrm{P}_{1}\right]-\mathrm{P}^{*}{ }_{1}=(1+\mu) \times \mathrm{P}_{0}-\left(1+\mathrm{R}^{*}\right) \times \mathrm{P}_{0}=\left(\mu-\mathrm{R}^{*}\right) \times \mathrm{P}_{0}$. Assuming the expected return is zero, 
$\operatorname{VaR}($ zero $)=-\left(\mathrm{P}^{*}{ }_{1}-\mathrm{P}_{0}\right)=-\mathrm{P}_{0} \mathrm{R}^{*}$. Next we can apply the transformation equation $k=(z-\mu) / \sigma$, with $z=-\mathrm{VaR}$ and $k=-k$, thus $\mathrm{VaR}=k \sigma-\mu(k-$ number corresponding to the confidence level $\alpha)$. Then it is $\mathrm{R}^{*}=-(k \sigma-\mu)$. By substituting $\mathrm{R}^{*}$ into the above equations, we have the following two formulas [8]: $\operatorname{VaR}($ zero $)=(k \sigma-\mu) \times \mathrm{P}_{0}$ and $\operatorname{VaR}($ mean $)=k \sigma \mathrm{P}_{0}$. If the confidence level $\alpha$ is given and the portfolio value is known, the only variable is the standard deviation of the rate of return, $\sigma$.

The tool that is extremely useful to manage risk is the component $\mathrm{VaR}$, which is a partition of the portfolio VaR that indicates the change of VaR if a given component was deleted. The component VaR measures the total contribution of component $i$ to the overall portfolio $\mathrm{VaR}$ and can uniquely be attributed to each of the individual components and aggregate linearly into the total diversified portfolio VaR. We can use it to have a risk decomposition of the current portfolio. The sum of individual VaRs is not so useful since it discards the diversification effects. Thus, we define the component $\mathrm{VaR}$ in term of marginal $\mathrm{VaR}(\Delta \mathrm{VaR})$ as follows [8]: Cmp_VaR $=\left(\Delta \mathrm{VaR}_{\mathrm{i}}\right) \times \mathrm{w}_{\mathrm{i}} \mathrm{P}=(\mathrm{VaR} / \mathrm{P}) \times \beta_{\mathrm{i}} \times \mathrm{w}_{\mathrm{i}} \mathrm{P}=\mathrm{VaR} \times\left(\beta_{\mathrm{i}} \mathrm{w}_{\mathrm{i}}\right)$. Note that the sum of all component VaRs (Cmp_VaR) is the VaR for the entire portfolio: $\Sigma_{(\mathrm{i}=1 ; n)} \mathrm{Cmp}_{-} \operatorname{VaR}_{\mathrm{i}}=\operatorname{VaR} \times\left(\sum_{(\mathrm{i}=1 ; \mathrm{n})} \beta_{\mathrm{i}} \mathrm{W}_{\mathrm{i}}\right)=\mathrm{VaR}$, where is: $\beta_{\mathrm{i}}=\sigma_{\mathrm{i}, \mathrm{P}} /\left(\sigma_{\mathrm{p}}\right)^{2}, \mathrm{w}_{\mathrm{i}}-$ weight defined by $\mathrm{w}_{\mathrm{i}}=\mathrm{P}_{\mathrm{i}} / \mathrm{P}, \mathrm{P}-$ portfolio value, $\mathrm{P}_{\mathrm{i}}-$ value of asset $i, \sigma_{\mathrm{i}, \mathrm{P}}-$ covariance between $\mathrm{i}^{\text {th }}$ asset ( $\mathrm{i}^{\mathrm{th}}$ type of ancillary service) and portfolio, $\left(\sigma_{\mathrm{p}}\right)^{2}-$ standard deviation for return rate of the portfolio. Intuitively the marginal risk of a portfolio with respect to an asset, is the increment in risk that obtained by buying a small amount of that asset. The marginal VaR is defined as the partial derivative with respect to the component weight. According to reference [8], it measure is defined to be the change in portfolio risk resulting from taking an additional dollar to a component: $\Delta \mathrm{VaR}_{\mathrm{i}}=k \times \sigma_{\mathrm{i}, \mathrm{P}} / \sigma_{\mathrm{p}}=k \times \sigma_{\mathrm{p}} \times \beta_{\mathrm{i}}=$ $(\mathrm{VaR} / \mathrm{P}) \times \beta_{\mathrm{i}}$. Individual VAR represents the VAR associated to individual component or asset constituting portfolio not taking into consideration diversification benefits. Individual VAR is calculated through the following equation: $\mathrm{VaR}_{\mathrm{i}}=k \sigma_{\mathrm{i}} \mathrm{P}_{\mathrm{i}}$, where $\sigma_{\mathrm{i}}$ represents the asset (type of ancillary service) volatility over past period.

\subsection{Conditional Value at Risk}

The general definition of Conditional Value at Risk (CVaR) is as follows. At this point, only the $\mathrm{CVaR}$ definition for continuous random variables will be given to create a more intuitive introduction into the topic. For continuous variable $\mathrm{X}$, the $\mathrm{CVaR}$ is the expected loss, conditional on the fact that the loss exceeds the $\mathrm{VaR}$ at the given confidence level.

Let $X$ be a continuous random variable representing loss. Given parameter $0<\alpha<1$, the $\alpha$-CVaR of $X$ is:

$$
\operatorname{CVaR}_{\alpha}(X)=\mathbf{E}\left[X \mid X \geq \operatorname{VaR}_{\alpha}(X)\right]
$$

Fundamental properties of CVaR, as a measure of risk with significant advantages over VaR, are derived for loss distributions in finance that can involve discreetness. Such distributions are of particular importance in applications because of the prevalence of models based on scenarios and finite sampling. $\mathrm{CVaR}$ is able to quantify dangers beyond $\mathrm{VaR}$, and moreover it is coherent. It provides optimization shortcuts which, through linear programming techniques, make practical many large-scale calculations that could otherwise be out of reach.

For continuous loss distributions, the $\mathrm{CVaR}$ at a given confidence level is the expected loss given that the loss is greater than the VaR at that level, or for that matter, the expected loss given that the loss is greater than or equal to the VaR. For distributions with possible discontinuities, however, it has a more subtle definition and can difer from either of those quantities, which for convenience in comparision can be designated by $\mathrm{CVaR}^{+}$and $\mathrm{CVaR}^{-}$, respectively. $\mathrm{CVaR}^{+}$has sometimes been called "mean shortfall", while "tail VaR" is a term that has been suggested for $\mathrm{CVaR}^{-}$. Generally [9]:

$\mathrm{CVaR}^{+}$(upper CVaR) is expected value of $X$ strictly exceeding VaR:

$$
\operatorname{CVaR}_{\alpha}^{+}(X)=\mathbf{E}\left[X \mid X>\operatorname{VaR}_{\alpha}(X)\right]
$$

$\mathrm{CVaR}^{-}$(lower $\left.\mathrm{CVaR}\right)$ is expected value of $X$ weakly exceedingVaR:

$$
\operatorname{CVaR}_{\alpha}^{-}(X)=\mathbf{E}\left[X \mid X \geq \operatorname{VaR}_{\alpha}(X)\right]
$$


$\mathrm{CVaR}$ is convex, but $\mathrm{VaR}, \mathrm{CVaR}^{-}, \mathrm{CVaR}^{+}$may be non-convex. The following inequalities are valid:

$$
\operatorname{VaR}_{\alpha}(X) \leq C \operatorname{VaR}_{\alpha}^{-}(X) \leq \operatorname{VVaR}_{\alpha}(X) \leq \operatorname{CVaR}_{\alpha}^{+}(X)
$$

Figure 1 shows the $\mathrm{VaR}$ and $\mathrm{CVaR}$ for a specific continuous random variable $\mathrm{X}$. The cumulative distribution function of $X$ can be used to find $\operatorname{VaR}_{\alpha}(X)$, and $\operatorname{VaR}_{\alpha}(X)$ can be used in turn to calculate $\mathrm{CVaR}_{\alpha}(\mathrm{X})$.
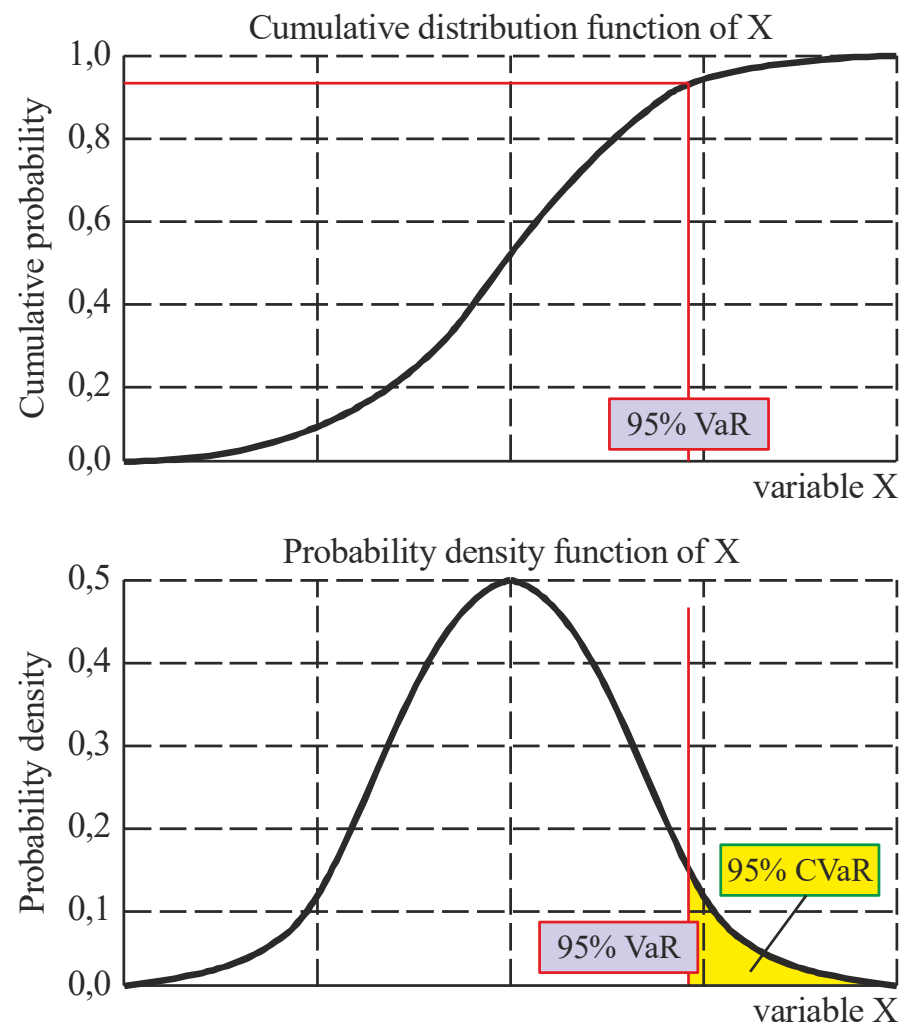

Figure $1 . \operatorname{VaR}_{\alpha}(\mathrm{X})$ and $\mathrm{CVaR}_{\alpha}(\mathrm{X})$ of a random variable $\mathrm{X}$ representing loss

\subsection{Conditional Value at Risk optimization theory}

Portfolio consists of $n$ instruments. Let $\mathbf{x}=\left(x_{1}, x_{2}, \ldots, x_{\mathrm{n}}\right)$ be a vector of positions, $\pi=\left(\pi_{1}, \pi_{2}, \ldots, \pi_{\mathrm{n}}\right)$ be a vector of initial prices, and $\mathbf{y}=\left(y_{1}, y_{2}, \ldots, y_{\mathrm{n}}\right)$ be a vector of uncertain prices in the next day. The loss function equals the difference between the current value of the portfolio $\left(x_{1} \pi_{1}+x_{2} \pi_{2}+\ldots+x_{\mathrm{n}} \pi_{\mathrm{n}}\right)$ and an uncertain value of the portfolio at the next day $\left(x_{1} y_{1}+x_{2} y_{2}+\ldots+x_{n} y_{n}\right)$ :

$$
\begin{aligned}
& f(\mathbf{x}, \mathbf{y})=\left(x_{1} \pi_{1}+x_{2} \pi_{2}+\ldots \quad\right. \text {, . . _ _ _ . . . . } \\
& \left.=x_{1}\left(\pi_{1}-y_{1}\right)+x_{2}\left(\pi_{2}-y_{2}\right)+\ldots \quad . . \quad n-y_{n}\right)
\end{aligned}
$$

If we do not allow short positios, the feasible set of portfolios is $n$-dimensional set of non-negative numbers: $\mathbf{X}=\left\{\left(x_{1}, x_{2}, \ldots, x_{\mathrm{n}}\right), x_{1} \geq 0, x_{2} \geq 0, \ldots, x_{\mathrm{n}} \geq 0\right\}$. Scenarios $\mathbf{y}^{\mathrm{j}}=\left(y_{1}^{\mathrm{j}_{1}}, y_{2}^{\mathrm{j}_{2}}, \ldots, y_{\mathrm{n}}^{\mathrm{j}}\right), \mathrm{j}=1,2, \ldots, \mathrm{J}$, are sample daily prices (historical data for $\mathrm{J}$ trading days).

CVaR minimization:

$$
\min _{\{\mathbf{x} \in \mathbf{X}\}} C V a R
$$


can be reduced to the following linear programming problem [10]:

$$
\min _{\left\{\mathbf{x} \in \mathbf{X}, \zeta \in \mathbf{R}, z \in \mathbf{R}^{J}\right\}} \zeta+v \sum_{j=1}^{J} z^{j}
$$

subject to

$$
z^{j} \geq f\left(\mathbf{x}, \mathbf{y}^{j}\right)-\zeta, \quad z^{j} \geq 0, \quad j=1,2, \ldots, J, \quad v=((1-\alpha) J)^{-1}=\text { const }
$$

By solving linear programming we find an optimal portfolio $\mathbf{x}^{*}$, corresponding VaR, which equals to the lowest optimal $\zeta^{*}$, and minimal $\mathrm{CVaR}$, which equals to the optimal value of the linear performance function.

Constraints $\mathbf{x} \in \mathbf{X}$ may account for various trading constraints, including mean return constraint (expected return should exceed 10\%). Similar to return-variance analysis, we can construct an efficient frontier and find a tangent portfolio. CVaR constraints in optimization problems can be replaced by a set of linear constraints. So,

$$
C V a R \leq C
$$

can be replaced by linear constraints:

$$
\begin{aligned}
\zeta+v \sum_{j=1}^{J} z^{j} & \leq C \\
& z^{j} \geq f\left(\mathbf{x}, \mathbf{y}^{j}\right)-\zeta, \quad z^{j} \geq 0, \quad j=1,2, \ldots, J, \quad v=((1-\alpha) J)^{-1}=\text { const }
\end{aligned}
$$

Loss distribution can be shaped using multiple CVaR constraints at different confidence levels in different times. The reduction of the CVaR risk management problems to linear programming is a relatively simple fact following from possibility to replace $\mathrm{CVaR}$ by some function $\mathrm{F}(\mathbf{x}, \zeta)$, which is convex and piecewise linear with respect to $\mathbf{x}$ and $\zeta[10]$.

\section{NUMERICAL RESULTS}

Calculation of the risk measures $\mathrm{VaR}$ and $\mathrm{CVaR}$ for a portfolio that contains more resources is more complex and in practice a more realistic task than calculating these risk measures for just one instrument. The following is the test case for calculating $\mathrm{VaR}$ and $\mathrm{CVaR}$ risk measures of such a portfolio, which is methodologically based on the modern Markowitz portfolio theory. This includes portfolio diversification elements, introducing a correlation matrix for all the individual instruments within the portfolio (in this case, the capacities of ancillary services for balancing of power system). In this test case, the system operator for functions balancing of power system has the capacities of secondary and tertiary reserves. Its portfolio, financially, is the revenues of 'up' and 'down' secondary control energy, the 'up' and 'down' tertiary control energy and the energy to secure the system's safety. So, in this case, the portfolio consists of five control resources (instruments). In the forthcoming period of 7 days (168 hours), the system operator has a portfolio of $815.000,0 \$$, with the parameters of each instrument as follows:

a. for secondary control energy 'up', $\mathrm{ASR}+=215.000,0 \$$;

b. for secondary control energy 'down', ASR- $=150.000,0 \$$;

c. for tertiary control energy 'up', $\mathrm{TR}+=375.000,0 \$$;

d. for tertiary control energy 'down', $\mathrm{TR}-=50.000,0 \$$;

e. for energy to secure the system's safety, TR-s $=25.000,0 \$$.

Over the past 7 days, the system operator has had the accepted hourly prices (historical data) for each type ancillary service (each instrument) as illustrated in Figure 2. For the analyzed test case and historical price data for each type of ancillary service in Figure 2, presented the results of the risk measures $\mathrm{VaR}$ and $\mathrm{CVaR}$ using Monte Carlo simulation. Monte Carlo simulation is based on the generation of random scenarios of prices for which the portfolio is revaluated. Looking at the hypothetical profits and losses under each scenario, it is possible to construct a histogram of expected profits and losses from which VaR is calculated. In this method we need a correlation and volatility matrix to generate the random scenarios. 
To perform Monte Carlo simulation it is necessary to have pricing models for all the instruments in our portfolio, and it is a procedure that is computationally intensive. The main advantage is that it is a forwardlooking assessment of risk, and it deals with options and non-linear position as we conduct a full valuation of the portfolio for each price scenario [6].

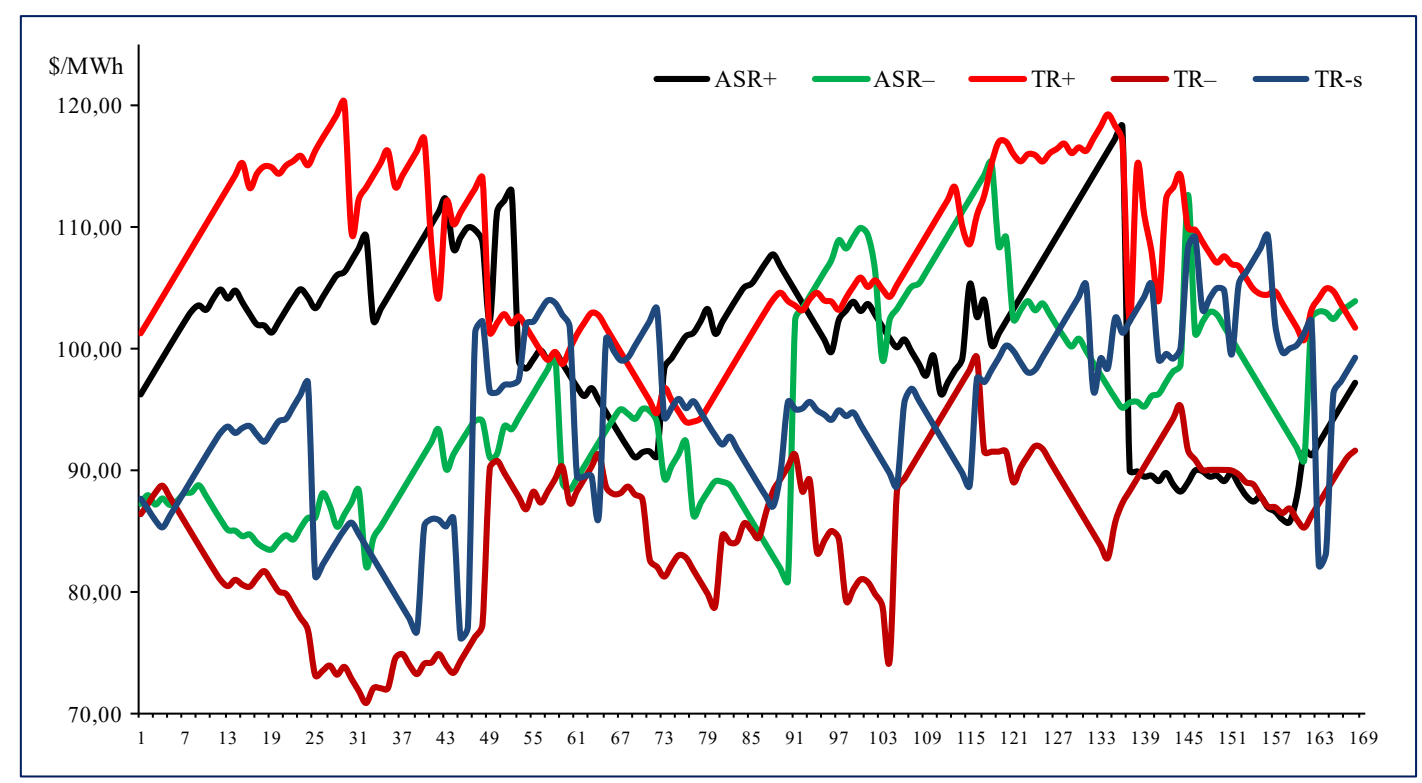

Figure 2. Hourly prices for different types of ancillary services

Table 1 and Table 2 show a summary of the risk results obtained for each single asset (each type of ancillary service) when held outside the portfolio. The computation of risk was performed with a confidence level of $\alpha=95 \%$ (number $k$ that corresponding to the confidence level $\alpha$ is $k=1,645$ ). Table 1 shows the results for standard deviation, individual VaR (Ind_VaR) and $\beta$-parameter for each type of ancillary service. The values of covariance matrix are given in Table $\overline{1}$.

Table 1. Standard Deviation for Analyzed Types of Ancillary Services, Their Individual VaR and $\beta$-Parameter, and Covariance Matrix

\begin{tabular}{|c|c|c|c|c|c|c|c|c|c|}
\hline Ancillary & Value of the & $\sigma$ & Ind_VaR & $\beta$ & \multicolumn{5}{|c|}{ Covariance Matrix } \\
\hline ASR+ & $215.000,0$ & 3,1232 & $11.044,82$ & 64,38 & 0,00098 & $-0,00037$ & 0,00034 & $-0,00072$ & $-0,00011$ \\
\hline ASR- & $150.000,0$ & 4,1057 & $10.129,80$ & 113,86 & $-0,00037$ & 0,00169 & 0,00067 & $-0,00043$ & 0,00091 \\
\hline $\mathrm{TR}+$ & $375.000,0$ & 3,2083 & $19.789,20$ & 140,18 & 0,00034 & 0,00067 & 0,00103 & $-0,00109$ & 0,00087 \\
\hline TR- & $50.000,0$ & 7,9955 & $6.575,74$ & $-92,67$ & $-0,00072$ & $-0,00043$ & $-0,00109$ & 0,00639 & $-0,00141$ \\
\hline TR-s & $25.000,0$ & 3,3218 & $1.365,97$ & 105,95 & $-0,00011$ & 0,00091 & 0,00087 & $-0,00141$ & 0,00110 \\
\hline
\end{tabular}

Table 2 shows the results for component VaR (Cmp_VaR) and marginal VaR $(\Delta \mathrm{VaR})$ for each type of ancillary service. The component $\mathrm{VaR}$ is given in percentage and in monetary units based on the portfolio $\mathrm{VaR}$ at the specified date. The values of correlation matrix are given in Table 2.

Table 2. Component and Marginal VaR for Analyzed Types of Ancillary Services and Correlation Matrix

\begin{tabular}{|c|c|c|c|c|c|c|c|c|}
\hline $\begin{array}{l}\text { Ancillary } \\
\text { Services }\end{array}$ & $\mathrm{Cmp}_{(\%)} \mathrm{VaR}$ & $\mathrm{Cmp}_{(\overline{\$})} \mathrm{VaR}$ & $\begin{array}{c}\Delta \mathrm{VaR} \\
(\$ / \$)\end{array}$ & \multicolumn{5}{|c|}{ Correlation Matrix } \\
\hline ASR+ & 16,983 & $1.438,78$ & 0,006692 & 1,00000 & $-0,28884$ & 0,33749 & $-0,28987$ & $-0,10457$ \\
\hline ASR- & 20,955 & $1.775,38$ & 0,011836 & $-0,28884$ & 1,00000 & 0,51363 & $-0,13186$ & 0,67309 \\
\hline $\mathrm{TR}+$ & 64,498 & $5.464,50$ & 0,014572 & 0,33749 & 0,51363 & 1,00000 & $-0,42832$ & 0,81758 \\
\hline TR- & $-5,685$ & $-481,65$ & $-0,009633$ & $-0,28987$ & $-0,13186$ & $-0,42832$ & 1,00000 & $-0,53458$ \\
\hline TR-s & 3,250 & 275,35 & 0,011014 & $-0,10457$ & 0,67309 & 0,81758 & $-0,53458$ & 1,00000 \\
\hline
\end{tabular}


The VaR of ancillary services portfolio can be understood as diversified VaR since we use this portfolio to reduce the overall risk for the balancing of power system. The diversification effect resulted in a total portfolio risk smaller then the sum of the single-ancillary service risks. The $95 \%$ individual VaR describing individual risk of each type of ancillary service in the portfolio is: $V a R_{i}=1,645 \sigma_{i} P_{i}$, where $\sigma_{i}$ represents the asset (one type of ancillary service) volatility over past period.

From the risk report given in Table 1 and Table 2 we can spot the difference of $40.433,17 \$$ between the undiversified portfolio $\mathrm{VaR}$ (sum of the individual $\mathrm{VaR}=48.905,53 \$$ ) and diversified portfolio VaR (sum of the component $\mathrm{VaR}=8.472,36 \$$ ) and the largest contribution of tertiary control energy 'up', TR+ to the overall portfolio VaR, where TR+ occupies the largest dollar position in ancillary services portfolio.

Marginal VaR is the largest for tertiary control energy 'up', TR+, secondary control energy 'down', ASR - and energy to secure the system's safety, TR-s. Therefore, in order to minimize portfolio VaR, we should cut them and/or reduce the positions. On the other hand, the exposure should be increased in case of secondary control energy 'up', ASR+ and tertiary control energy 'down', TR- which display the lowest marginal VaR. Both suggestions find the solution in a form of derived new holdings that would reduce the portfolioVaR. The best and more practical interpretation of the marginal VaR calculated for all positions in the portfolio would be: the higher $\Delta \mathrm{VaR}_{\mathrm{i}}$ the corresponding exposure of the $\mathrm{i}^{\text {th }}$ component should be reduced to lower the overall portfolio VaR.

For this portfolio $\mathrm{VaR}$ and $\mathrm{CVaR}$ risk measures after 24 hours following next week (next 168 hours), with a $95 \%$ of confidence level are given in Table 3.

Table 3. Indices VaR and CVaR for Analyzed Ancillary Services Portfolio

\begin{tabular}{lccccc}
\hline \multirow{2}{*}{ Risk measure } & \multicolumn{2}{c}{ Absolute CVaR } & risk measure & \multicolumn{2}{c}{ CVaR relative to mean } \\
& Returns (\%) & portfolio value $(\$)$ & & Returns (\%) & portfolio value $(\$)$ \\
\hline VaR & $-1,040$ & $-8.472,36$ & VaR & $-0,977$ & $-7.959,45$ \\
$\mathrm{CVaR}^{-}$ & $-6,012$ & $-48.998,90$ & $\mathrm{CVaR}^{-}$ & $-5,949$ & $-48.486,00$ \\
$\mathrm{CVaR}^{+}$ & $-6,399$ & $-52.153,66$ & $\mathrm{CVaR}^{+}$ & $-6,336$ & $-51.640,76$ \\
$\mathrm{CVaR}^{+}$ & $-6,633$ & $-54.064,72$ & $\mathrm{CVaR}^{+}$ & $-6,571$ & $-53.551,81$ \\
\hline
\end{tabular}

We can find the portfolio that minimises the CVaR measure (a feature that is only possible into the CVaR framework) for the specified CVaR horizon. The optimization results are given in Table 4. It is important to note that the optimal value of the ancillary services portfolio should take into account the technical parameters of certain types of ancillary services, which are in correlation with the economic parameters of the portfolio structure. In order to avoid cases that any service has a value of $0 \$$, which means that it is excluded from balancing of power system, it is necessary to introduce additional technical and economic constraints in the model given in point 2.3. Technical and economic parameters for the ancillary services of the same type with opposite direction of action (ASR+ and ASR-, or TR + and TR-), as well as the ancillary services of different type with complementary direction of action (ASR + and TR + , or ASRand TR-), can easily be incorporated in the model through additional constraints. This example clearly illustrates that portfolio of ancillary services can be efficiently optimized to ensure power system security and service deliverability.

Table 4. Optimal Portfolio that Minimizes the CVaR

\begin{tabular}{ccc}
\hline Ancillary Services & Value of the Service $(\$)$ & Optimal Value of the Service $(\$)$ \\
\hline ASR+ & $215.000,0$ & $232.305,28$ \\
ASR- & $150.000,0$ & $113.700,13$ \\
TR+ & $375.000,0$ & $274.233,87$ \\
TR- & $50.000,0$ & $173.425,03$ \\
TR-s & $25.000,0$ & $21.343,28$ \\
total $=$ & $815.000,0$ & $815.007,60$ \\
VaR (\$) & $-8.472,36$ & $-7.367,74$ \\
VaR (\%) & $-1,040$ & $-0,904$ \\
CVaR (\$) & $-52.153,66$ & $-7.811,93$ \\
CVaR (\%) & $-6,399$ & $-0,958$ \\
\hline
\end{tabular}

\section{CONCLUSION AND FUTURE RESEARCH}

This paper focuses on the assessment and analysis of the risk of balancing the power system related to providing ancillary services. Risk on ancillary services portfolio is modeled through Value at Risk and Conditional Value at Risk measures. When considering portfolio in context of ancillary services with many instruments it can be hard to find the optimal portfolio mixture, which minimizes Value at Risk-VaR, since 
$\mathrm{VaR}$ is not a convex function with respect to the portfolio weights. An alternative and better risk measure is Conditional Value at Risk-CVaR, which is the expected loss given that the loss exceeds some threshold. $\mathrm{CVaR}$ is a convex function of the portfolio weights and is therefore easier to optimize. Risk control can be accomplished by two means: hedging which is a technique to offset particular source of risk and diversification. In an electricity market, forward contracts which omit the risk of price volatility are available for hedging against risk whereas a variety of spot markets such as automatic generation control and reserve markets are provided for making diversification in the trading plan.

Mainly two topics for future research are important. First, we have assumed that the random variables are continuously distributed. However, the given results could be wrong for random variables with a discrete probability distribution. So the question arises what can be said about marginal risk contributions if random variables are not continuously distributed. This is an important issue because all real-world probability distributions are in fact discrete distributions. Second, the effect of correlation between assets has not been implemented in the used model/scenario, but it is rather easy to apply, and surely can have large effect on the outcome. Constraints using correlation coefficients of assets with strong positive correlation could be used in the optimization problem to encourage higher amount of negatively correlated assets.

\section{REFERENCES}

[1] S. C. Schnabel, H. J. Appelrath, S. Lehnhoff, M. Troschel, "A risk management for agent-based control of ancillary service provision from distributed energy resources in smart grids," Proceedings of the 28th Enviro Info 2014 Conference, Oldenburg, Germany, 2014.

[2] J. Doege, M. Fehr, J. Hinz, H. J. Luethi, M. Wilhelm, "On value of flexibility in energy risk management," Concepts, models, solutions, 2006.

[3] S. Fiorenzani, "Quantitative methods for electricity trading and risk management: advanced mathematical and statistical methods for energy finance," Palgrave Macmillan, 2006.

[4] M. Liu, F. F. Wu, "A survey on risk management in electricity markets," Proceedings IEEE Power Engineering Society General Meeting, Montreal, Canada, 2006.

[5] R. Dahlgren, C. C. Liu, J. Lawarree, "Risk assessment in energy trading," IEEE Transactions on Power Systems, vol. 18, no. 2, pp. 503-511, 2003.

[6] C. Blanco, "Value-at-Risk of energy: Is VaR useful to manage energy price risk," Commodities, pp. 62-71, 1998.

[7] P. Richtárik, "Optimization methods in finance," lectures notes, 2015.

[8] D. Bo, "Value at Risk," Undergraduate Research Opportunity Programme in Science, Department of Mathematics, National University of Singapore, 2001.

[9] P, Jorion, Value at Risk, McGraw-Hill, 2nd edition, 2001.

[10] R. T. Rockafellar, S. P. Uryasev, "Optimization of Conditional Value-at-Risk," Journal of Risk, vol. 2, pp. 21-42, 2000.

\section{BIOGRAPHIES OF AUTHORS}

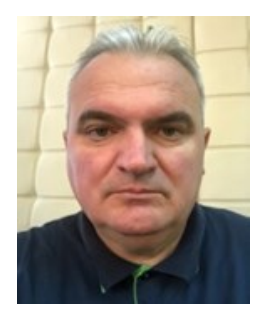

Omer Hadzic received the degree of Electrical Engineer in 1991 and MSc degree in 2011 from the Faculty of Electrical Engineering, University of Sarajevo, Bosnia and Herzegovina. He is advisor to the board and general manager at Independent System Operator in Bosnia and Herzegovina. His areas of interest include operation, planning and economics of power systems.

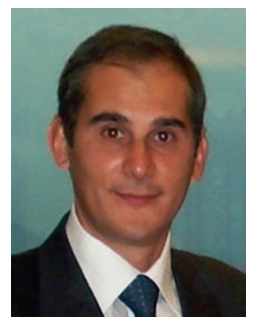

Smajo Bisanovic received the degree of Electrical Engineer in 1991, MSc degree in 1994 and PhD degree in 2009 from the Faculty of Electrical Engineering, University of Sarajevo, Bosnia and Herzegovina. He is associate professor at the Faculty of Electrical Engineering, University of Sarajevo, Bosnia and Herzegovina and head of control and trading department at Public Enterprise Elektroprivreda of Bosnia and Herzegovina d.d. - Sarajevo. His areas of interest include operation, planning and economics of power systems and application of reliability theory to power systems. 